\title{
Fatores de risco e comorbidades associados aos casos de tuberculose notificados no município de Campo Grande, Mato Grosso do Sul
}

\author{
Risk factors and comorbidities associated with notified cases of tuberculosis in the \\ municipality of Campo Grande, Mato Grosso do Sul
}

Factores de riesgo y comorbilidades asociados a casos notificados de tuberculosis en el municipio de Campo Grande, Mato Grosso do Sul

João Pedro Arantes da Cunha ${ }^{1 *}$, Ana Maria Campos Marques ${ }^{1}$, Rachel Carvalho Lemos ${ }^{1}$, Paloma Almeida Kowalski ${ }^{1}$, Thiago Vilela de Freitas ${ }^{1}$, Emily Ruiz Cavalcante ${ }^{1}$, Rafael Vilela de Campos ${ }^{2}$.

\section{RESUMO}

Objetivo: Estimar a incidência e descrever as comorbidades de todos os casos de tuberculose notificados ao Sistema Nacional de Agravos de Notificação (SINAN) do município de Campo Grande/MS. Métodos: Tratase de uma pesquisa transversal de dados secundários que analisou todos os 2806 casos notificados ao Sistema Nacional de Agravos de Notificação no município de Campo Grande/MS, período de janeiro de 2014 a dezembro de 2019. Resultados: A taxa de incidência no município variou no período de 31,56 a 51,42 casos/100 mil habitantes. Observou-se predomínio no sexo masculino $(80,3 \%)$. Quanto às comorbidades, $70,5 \%$ apresentaram pelo menos uma. O tabagismo foi o agravo mais prevalente (27\%), seguido pelo uso de substâncias psicoativas, álcool e coinfecção pelo vírus HIV/Aids (13,8\%). O diabetes foi a doença menos prevalente com 5,4\%, o que difere das taxas nacionais. A modalidade de Tratamento Diretamente Observado foi realizada em 3,8\% dos casos, taxa muito inferior a nacional. Conclusão: Evidenciou-se a possível ocorrência de atraso do diagnóstico. Um terço dos casos de tuberculose foram diagnosticados nos hospitais. O coeficiente de incidência no município foi superior ao do estado e do Brasil, principalmente nos anos de108 e 2019, com uma taxa de incidência de 51,42 e 41,6, respectivamente.

Palavras-chave: Tuberculose, Epidemiologia, Comorbidade, Tabagismo, Alcoolismo.

\section{ABSTRACT}

Objective: To estimate and describe the comorbidities of all tuberculosis cases reported to the National System of Notifiable Diseases (SINAN) in the municipality of Campo Grande/MS. Methods: This is a crosssectional survey of secondary data that analyzed all 2806 cases reported to the National System of Notifiable Diseases in the city of Campo Grande/MS, from January 2014 to December 2019. Results: The incidence rate in the municipality it ranged in the period from 31.56 to 51.42 cases/100 thousand inhabitants. There was a predominance of males $(80.3 \%)$. As for comorbidities, $70.5 \%$ had at least one. Smoking was the most prevalent health problem (27\%), followed by the use of psychoactive substances, alcohol and HIV/Aids coinfection (13.8\%). Diabetes was the least prevalent disease with $5.4 \%$, which differs from national rates. The Directly Observed Treatment modality was performed in 3.8\% of the cases, a much lower rate than the national rate. Conclusion: The possible occurrence of delayed diagnosis was evidenced. A third of TB cases were diagnosed in hospitals. The incidence coefficient in the city was higher than in the state and in Brazil, mainly in the years 108 and 2019, with an incidence rate of 51.42 and 41.6 , respectively.

Keywords: Tuberculosis, Epidemiology, Comorbidity, Smoking, Alcoholism.

1 Universidade Estadual de Mato Grosso do Sul (UEMS), Campo Grande - MS.

*E-mail: jparantesdacunha@gmail.com

2 Universidade para o Desenvolvimento do Estado e da Região do Pantanal (Uniderp), Campo Grande - MS. 


\section{RESUMEN}

Objetivo: Estimar la incidencia y describir las comorbilidades de todos los casos de tuberculosis notificados al Sistema Nacional de Enfermedades Notificables (SINAN) en la municipio de Campo Grande/MS. Métodos: Se trata de una encuesta transversal de datos secundarios que analizó los 2806 casos notificados al Sistema Nacional de Enfermedades Notificables en la ciudad, de enero de 2014 a diciembre de 2019. Resultados: La tasa de incidencia se osciló en el período de 31,56 a 51,42 casos / 100 mil habitantes. Predominó el sexo masculino $(80,3 \%)$. En cuanto a las comorbilidades, el $70,5 \%$ tenía al menos una. El tabaquismo fue el problema de salud más prevalente (27\%), seguido del uso de sustancias psicoactivas, alcohol y coinfección por VIH / SIDA (13,8\%). La diabetes fue la enfermedad menos prevalente con un $5,4 \%$, que difiere de las tasas nacionales. Conclusión: Se evidenció la posible ocurrencia de diagnóstico tardío. Un tercio de los casos de tuberculosis se diagnosticaron en hospitales. El coeficiente de incidencia en la ciudad fue mayor que en el estado y en Brasil, principalmente en los años 108 y 2019, con una tasa de incidencia de 51,42 y 41,6, respectivamente.

Palabras clave: Tuberculosis, Epidemiología, Comorbilidad, Tabaquismo, Alcoholismo.

\section{INTRODUÇÃO}

A tuberculose (TB) permanece entre as doenças infectocontagiosas mais prevalentes em todo o mundo. Um terço da população global encontra-se infectada pelo Mycobacterium tuberculosis, com uma incidência anual estimada em cerca de 8,8 milhões de casos (OLIVEIRA MG, et al., 2014). No ano de 2019, cerca de 1,2 milhão de indivíduos morreram e dez milhões apresentaram a doença. O Brasil segue entre os 30 países de carga elevada de TB e da coinfecção TB-HIV, considerado prioritário pela Organização Mundial de Saúde (OMS) para o controle da doença no mundo (OMS, 2020).

O coeficiente de incidência da TB no país aumentou nos anos de 2017 e 2018 em relação aos períodos anteriores. Nesse período houve declínio na incidência entre idosos, especificamente em indivíduos acima de 65 anos, assim como foi observado aumento em crianças, menores de 10 anos, e na faixa etária de 10 a 64 anos. Em 2019 foi de 35 casos/100 mil habitantes, uma incidência de 73.864 casos novos (MINISTÉRIO DA SAÚDE, 2017; MINISTÉRIO DA SAÚDE, 2020).

A forma clínica mais frequente é a TB pulmonar, a que mantém a cadeia de transmissão, um doente com a forma pulmonar positiva durante 1 ano poderá infectar, em média, de 10 a 15 pessoas, estima-se que 10\% adoeçam: $5 \%$ nos dois primeiros anos da infecção e $5 \%$ ao longo da vida persistindo o risco de adoecimento por TB por toda a vida (MINISTÉRIO DA SAÚDE, 2019b).

Os fatores que facilitam o contágio são: contato intradomiciliar com os doentes com a forma pulmonar bacilífera e a intensidade do contato, portanto o diagnóstico precoce das formas pulmonares e o tratamento dos doentes são aspectos centrais no controle da tuberculose, na maioria dos serviços de saúde, o diagnóstico da patologia é tardio e a confirmação da positividade ocorre em menos de $50 \%$ de todos os casos ativos (ORME IM, et al., 2015; AMICOSANTE MA, et al., 2017).

Dentre os fatores facilitadores da transmissão e da progressão para a doença temos os fatores endógenos, a integridade do sistema imune e as comorbidades, como: etilismo, diabetes mellitus, desnutrição, coinfecção pelo HIV, uso de drogas lícitas e ilícitas. Logo, é importante identificar as comorbidades a fim de assegurar o diagnóstico precoce a esses pacientes (OLIVEIRA MG, et al., 2014).

Com o advento da AIDS, no início da década de 1980, o cenário da TB se intensificou. A coinfecção vem se expandindo, alterando a configuração prognóstica e epidemiológica da doença. Para indivíduos com diagnóstico de HIV, o risco de desenvolver tuberculose é de $10 \%$ ao ano, enquanto para pacientes isentos do vírus, o percentual é de 10\% ao longo da vida (GASPAR RS, et al., 2016; MINISTÉRIO DA SAÚDE, 2018a).

Em relação à associação com diabetes mellitus (DM), os indivíduos possuem maior risco de desenvolver a tuberculose ativa. Além do risco de falência ao tratamento especifico da TB com maior risco de óbito. A probabilidade de adoecimento por TB é de 2,4 a 8,3 vezes mais do que nos não diabéticos (OROFINO RL, et 
al., 2012; SILVA DR, et al., 2018). O ato de fumar eleva em mais de 2,5 vezes o risco de contrair TB, aumentando, consequentemente, a taxa de mortalidade. Estima-se que, em todo o mundo, 1,3 bilhão de pessoas utilizam tabaco, a maioria habita países subdesenvolvidos ou em desenvolvimento. A mortalidade cai significativamente com a interrupção do tabagismo, em média $65 \%$ em relação aos que permanecem fumantes (SILVA DR, et al., 2018).

Considerando que a busca ativa de casos de TB entre as populações de risco de adoecimento é um dos pilares para diminuir a incidência da doença, a presente pesquisa teve por objetivo descrever o perfil epidemiológico dos pacientes diagnosticados com tuberculose e estimar a incidência de fatores de risco, agravos e doenças associadas de todos os casos notificados pelos serviços de saúde no período de 2014 a 2019.

\section{MÉTODOS}

Estudo descritivo de dados secundários, restrospectivo, quantitativo e transversal de todos os casos, diagnosticados, notificados e registrados no período de 01 de janeiro de 2014 a 31 de dezembro de 2019. Os dados foram obtidos a partir do Sistema Nacional de Agravos de Notificação (SINAN), fornecidos pela Secretaria de Saúde de Campo Grande, MS (SESAU/CG-MS) na forma de banco de dados Excel.

As variáveis analisadas incluíram: sexo, idade, unidade de saúde notificadora, tipo de entrada, etnia, formas clínicas pulmonar e extrapulmonar, exames, doenças e agravos: diabetes, tabagismo, coinfecção por HIV, uso de substâncias psicoativas, alcoolismo. Foram avaliados o local dos diagnósticos, sistema prisional, hospitais e unidades de saúde do município.

Para a análise de dados utilizou-se o programa Bioestat 5.0. Para o cálculo do coeficiente de incidência utilizou-se o total de casos de casos $/ 100$ mil habitantes e base populacional do Instituto Brasileiro de Geografia e Estatística (IBGE). Projeto aprovado pelo Comitê de Ética em Pesquisa/ Conselho Nacional de Ética em Pesquisa (CEP/CONEP) no 3.951.339/ abril de 2020. Foi utilizado checklist Strengthening The Reporting of OBservational Studies in Epidemiology (STROBRE) ara estudos observacionais e transversais em epidemiologia para avaliação do mérito da pesquisa.

\section{RESULTADOS}

No período foram notificados 2806 casos de tuberculose no município, sendo que 2062 (73,5\%) casos novos com coeficiente de incidência variou de 31,56 a 51,42/100.000 habitantes por ano (Tabela 1).

Tabela 1 - Distribuição do coeficiente de Incidência por ano de todos os casos novos de TB notificados no município de Campo Grande/MS de 2014 a 2019.

\begin{tabular}{ccc}
\hline Ano & N & Coeficiente de Incidência/100.000 Habitantes \\
\hline 2014 & 301 & 33,21 \\
2015 & 286 & 31,56 \\
2016 & 315 & 34,76 \\
2017 & 317 & 34,98 \\
2018 & 466 & 51,42 \\
2019 & 377 & 41,6 \\
\hline
\end{tabular}

Legenda: $\mathrm{N}$ = número de casos, \% = porcentagem de casos. Fonte: Cunha JPA, et al., 2021; dados extraídos e calculados de IBGE, 2021.

Eram do sexo masculino 2254 (80,3\%), enquanto 552 eram do sexo feminino (19,7\%). Os menores de 10 anos corresponderam a 44 casos (1,6\%), enquanto $113(4 \%)$ eram entre 10 e 19 anos, $2358(84 \%)$ entre 20 e 59 anos e 291 casos em maiores de 60 anos (10,4\%). Quanto à etnia declarada, 1468 eram pardos (52,3\%), $667(23,8 \%)$ brancos, 229 pretos (8,2\%), 35 amarelos (1,2\%), 13 indígenas (0,5\%), 394 ignorados e vazios (14\%) (Tabela 2). 
Tabela 2 - Distribuição de todos os casos de tuberculose notificados ao SINAN pelo município de Campo Grande/MS no período de 2014 a 2019 de acordo com o sexo e grupo etário.

\begin{tabular}{lcc}
\hline Variáveis & $\mathbf{N}$ & $\%$ \\
\hline Total de casos & $\mathbf{2 8 0 6}$ & $\mathbf{1 0 0}$ \\
\hline Sexo & 552 & $19,7 \%$ \\
\hline Feminino & 2254 & $80,3 \%$ \\
Masculino & & $1,6 \%$ \\
\hline Faixa etária & 44 & $4 \%$ \\
\hline$<10$ anos & 113 & $84 \%$ \\
10 a 19 anos & 2358 & $10,4 \%$ \\
\hline 60 anos 59 anos & 117 & \\
\hline
\end{tabular}

Legenda: $\mathrm{N}$ = número de casos, \% = porcentagem de casos. Fonte: Cunha JPA, et. al., 2021.

Os serviços notificadores foram: Atenção Primária/Secundária, Rede Hospitalar e Sistema Prisional. As Unidades de Atenção Básica notificaram 1245 casos (44,4\%), a Rede Hospitalar 865 (30,8\%) e o sistema prisional $696(24,8 \%)$ do total de casos (Tabela 3). A forma clínica pulmonar predominou, com 2342 casos (83,5\%), sendo 1218 com baciloscopia positiva, $52 \%$ dos casos pulmonares.

Tabela 3 - Distribuição do número e percentual de todos os casos de TB diagnosticados e notificados, segundo os diferentes Serviços de Saúde do município no período de 2014 a 2019.

\begin{tabular}{lcc}
\hline Unidades de saúde de notificação & $\mathbf{N}$ & $\%$ \\
\hline Atenção Primária e Secundária & 1245 & $44,4 \%$ \\
Rede Hospitalar & 865 & $30,8 \%$ \\
Sistema Prisional & 696 & $24,8 \%$ \\
Total de casos & 2806 & $100 \%$ \\
\hline
\end{tabular}

Legenda: $\mathrm{N}$ = número de casos, \% = porcentagem de casos. Fonte: Cunha JPA, et. al., 2021.

Dos $1984(70,5 \%)$ casos que apresentavam pelo menos uma comorbidade, o tabagismo foi o agravo mais prevalente, com 757 casos (27\%), seguido do uso de substâncias psicoativas, com 608 (21,7\%), e pelo etilismo com $582(20,7 \%)$. A segunda associação mais comum foi o alcoolismo e usuários de substâncias psicoativas ilícitas, seguido pela coinfecção TB-HIV. Dentre os usuários de drogas ilícitas, $84(13,8 \%)$ eram HIV positivo (Tabela 4).

Tabela 4 - Distribuição do número e percentual dos casos de TB pulmonar positiva diagnosticados e notificados pelo município no período de 2014 a 2019 segundo o número de comorbidades e agravos associados.

\begin{tabular}{lcccc}
\hline \multirow{2}{*}{ Comorbidades } & \multicolumn{2}{c}{ Total de casos } & \multicolumn{2}{c}{ Pulmonar Positiva } \\
\cline { 2 - 5 } & $\mathrm{N}$ & $\%$ & $\mathrm{~N}$ & $\%$ \\
\hline 1 comorbidade & & & & \\
\hline Tabagismo & $757 / 2806$ & $27 \%$ & $666 / 757$ & $88 \%$ \\
Etilismo & $582 / 2806$ & $20,7 \%$ & $436 / 582$ & $79,1 \%$ \\
Substâncias psicoativas & $608 / 2806$ & $21,7 \%$ & $548 / 608$ & $90,1 \%$ \\
HIV/AIDS & $356 / 2806$ & $13,8 \%$ & $209 / 387$ & $54 \%$ \\
Diabetes & $151 / 2806$ & $5,4 \%$ & $134 / 151$ & $88,7 \%$ \\
Total & 2806 & & & \\
\hline 2 ou mais comorbidades associados & & & \\
\hline Dependência Química, Alcoolismo e Tabagismo & $172 / 2806$ & $6,1 \%$ & $149 / 172$ & $86,6 \%$ \\
Usuários de Substâncias Psicoativas em etilistas & $256 / 582$ & $44 \%$ & $203 / 256$ & $79,3 \%$ \\
Usuários de substâncias psicoativas & $84 / 608$ & $13,8 \%$ & $53 / 84$ & $63,1 \%$ \\
em HIV positivos & & & & \\
\hline
\end{tabular}

Legenda: $\mathrm{N}=$ número de casos, \% = porcentagem de casos. Fonte: Cunha JPA, et al., 2021. 
A quarta comorbidade mais prevalente foi coinfecção pelo vírus HIV/Aids, $387(13,8 \%)$. O teste rápido para HIV foi realizado no momento do diagnóstico da TB para 2315 casos $(82,5 \%)$ (Tabela 5). O diabetes foi a menos prevalente, com 151 casos $(5,4 \%)$ e $236(11,3 \%)$.

Tabela 5 - Distribuição do número e percentual de casos de TB diagnosticados e notificados pelo município no período de 2014 a 2019, segundo o diagnóstico pré ou pós HIV/Aids.

\begin{tabular}{lcc}
\hline Variáveis & $\mathbf{N}$ & $\%$ \\
\hline Diagnóstico prévio de HIV & 356 & $92 \%$ \\
Novo diagnóstico de HIV em conjunto com a TB & 81 & $8 \%$ \\
Total & 387 & $100 \%$ \\
\hline Método de diagnóstico & & \\
\hline Teste rápido de HIV/Aids realizado no momento do diagnóstico de TB & 2315 & 82,5 \\
Não foi feito rastreio & 491 & 17,5 \\
Total de casos & 2806 & 100 \\
\hline
\end{tabular}

Legenda: $\mathrm{N}$ = número de casos, \% = porcentagem de casos. Fonte: Cunha JPA, et. al., 2021.

Dos 2179 casos notificados de TB pulmonar 77,6\% realizaram baciloscopia de escarro, destes, 1248 $(57,3 \%)$ tinham baciloscopia positiva e $524(24 \%)$ negativa. Em 1557 casos de forma pulmonar cultura para BK foi realizada em $71,45 \%$ com $869(55,81 \%)$ resultados positivos. O teste molecular TRM/TB foi realizado em 742 casos $(26,4 \%)$, resultado positivo em $595(80,2 \%)$.

\section{DISCUSSÃO}

No período do estudo, houve um predomínio de casos de TB no sexo masculino, com o total de 2254 casos $(80,3 \%)$. A tuberculose atinge majoritariamente indivíduos deste gênero, tendo os homens duas vezes mais chance de adoecer, esses índices encontrados foram superiores ao da literatura. A maioria dos estudos evidenciam um percentual de 60-70\%. Segundo a OMS a relação entre homem/mulher oscila entre 1,5:1 a 2,1:1, encontramos 4:1. Esses dados podem ser justificados por diversos fatores econômicos, sociais e culturais. Homens são mais propensos a viver em situação de rua e privados de liberdade, serem tabagista, alcoólicos e usuários de substâncias ilícitas, agravos que aumentam a incidência da doença (FREITAS VMT, et al., 2016).

Do total de casos notificados no período, 696 (26,9\%) foram dos presídios, destes 685 (98,4\%) do sexo masculino e 11 do sexo feminino $(1,6 \%)$. Isso reafirma o confinamento e gênero como fator de risco para a incidência de TB (GASPAR RS, et al., 2016; FREITAS VMT, et al., 2016).

Segundo informações do Ministério da Saúde (2019b), homens entre 25 e 40 anos adoecem mais por tuberculose do que as mulheres, fato que pode estar relacionado ao estilo de vida. Este foi o grupo etário de maior incidência, perfazendo mais de $60 \%$ da população do estudo. A distribuição por grupo etário segue a nacional, demonstrando um predomínio de acometimento no grupo etário de 20 e 39 anos, uma das fases mais ativas da vida (FREITAS VMT, et al., 2016; OLIVEIRA LGF, et al., 2021).

A TB possui relação com as condições de higiene e de saneamento básico da população, com a classe social e sobretudo com aglomerações populacionais. No país, os casos são notificados em sua maioria nas regiões de periferia, onde ocorre maior aglomeração. Em Campo Grande não é diferente, considerando que um número representativo dessa doença é registrado na população carcerária, correspondendo a $24,8 \%$ do total de diagnósticos (FREITAS VMT, et al., 2016).

Além dos fatores ambientais, os aspectos comportamentais presentes nas penitenciárias favorecem a um risco superior de infecção. A maioria dos detentos possuem antecedentes de má nutrição e de uso de tabaco, álcool e outras drogas ilícitas. Além disso, uma vez na prisão mantém os comportamentos de risco, como uso de drogas e sexo sem proteção, sendo ambos fatores que contribuem para a infecção pelo HIV (VALENÇA MS, et al., 2016).

A maioria dos diagnósticos (44,4\%) ocorreram na Atenção Primária, que é a porta de entrada para os pacientes com suspeita de TB, onde se realiza medidas de prevenção, de promoção da saúde e diagnóstico 
precoce dos sintomáticos respiratórios, de grupos de risco e suas comorbidades, além do tratamento e acompanhamento dos casos.

Com os dados do período de 2014 a 2019 obtidos no município, estima-se que um terço dos casos de TB $(30,8 \%)$ estão sendo diagnosticados nos hospitais, possivelmente em estados avançados ou com sinais de gravidade, seja pela própria TB ou pelas doenças associadas apontando que a busca ativa de casos pela Atenção Primária do município não atinge a meta de $80 \%$ recomendada pelo Ministério da Saúde para o Controle da TB no país (MINISTÉRIO DA SAÚDE, 2019b).

Em 2019, no Brasil, houve um aumento na proporção de casos diagnosticados e acompanhados por serviços da APS. Cerca de $54,0 \%$ dos casos novos foram notificados e $61,4 \%$ foram acompanhados na Atenção Primária, não alcançando o suficiente para alcançar as metas de aumentar o diagnóstico precoce de casos novos, com consequente diminuição da incidência da doença (MINISTÉRIO DA SAÚDE, 2019b).

Indivíduos com comorbidades, HIV/AIDS, drogas, tabaco, alcoolismo, diabetes e doenças mentais, são os com maior propensão a adoecer por TB. De todos os casos notificados no município, $80 \%$ dos doentes apresentavam, no momento do diagnóstico, pelo menos uma comorbidade (MINISTÉRIO DA SAÚDE, 2019b).

O uso associado do tabaco representou uma incidência de $27 \%$ de todos os casos notificados durante o período no município, sendo a associação mais frequente. Existe forte relação entre TB e consumo de álcool, tabaco e drogas ilícitas, sendo o tabagismo mais expressivo. Considera-se a maneira de se obter a substância ativa, através da combustão do tabaco e a consequente inalação da fumaça o papel fundamental para criar condições de desenvolvimento da TB no fumante, desde a perpetuação de uma disfunção ciliar, ocasionando uma resposta imune reduzida, a defeitos na resposta imune de macrófagos, ocorrendo com ou sem uma diminuição da contagem de CD4. Esses são alguns dos fatores estudados que justificam o aumento da suscetibilidade à infecção por Mycobacterium tuberculosis (SILVA VD, et al., 2017; NOVOTNY T, et al., 2017).

Esses fatores elevam o risco de persistência do bacilo após o tratamento e o risco de infecção latente pela TB (ILTB), bem como na progressão da TB em atividade, menor adesão ao tratamento e à interferência na coleta do exame de escarro, predispondo falsos-negativos. Esses dados associados aos resultados encontrados evidenciam a necessidade de ações de redução das taxas de tabagismo pelos pacientes com TB (NOVOTNY T, et al., 2017).

Conforme exposto por alguns autores, aproximadamente $15 \%$ dos casos de tuberculose em todo o mundo podem estar ligados à diabetes mellitus (DM). De acordo com os dados observados no estudo apenas $5 \%$ dos pacientes no período deste estudo tinham essa comorbidade, sendo uma incidência discordante com a literatura, induzindo a hipótese de subdiagnóstico, falha no preenchimento ou anamnese incompleta do paciente (SILVA DR, et al., 2018).

O diabetes mellitus é uma importante situação de risco, pois indivíduos que apresentam estas duas condições tendem a desenvolver taxas elevadas de falência do tratamento para TB, bem como maior risco de óbito, designando-se como um grupo de risco para desenvolver resistência às drogas utilizadas além da TB poder provocar intolerância à glicose e dificultar assim o controle glicêmico de pacientes com DM. Para o controle global da doença, a associação entre DM e TB representa um desafio oportuno e crescente (PEREIRA SM, et al., 2016).

A coinfecção por HIV ocorreu em $12,7 \%$ dos casos, sendo a quarta comorbidade mais frequente. Atualmente, a TB permanece como a principal causa de óbito em indivíduos portadores de HIV, sendo responsável por cerca de uma em cada três mortes relacionadas à AIDS. Estima-se que o indivíduo acometido pelo vírus apresenta 28 vezes a mais de contrair a TB em comparação a indivíduos sem a doença (MINISTÉRIO DA SAÚDE, 2018a).

No ano de 2019, cerca de 10 milhões de pessoas contraíram TB no mundo, sendo que $9 \%$ estavam eram HIV positivos, enquanto 1,7 milhões adquiriram HIV. No país, a estimativa da coinfecção em 2016 foi de 9,4\%. Isto significa que dos 69 mil novos casos de TB notificados no ano, 6,5 mil testaram positivo para o vírus. Estima-se, ainda, que $44 \%$ das pessoas que vivem com HIV e tuberculose não sabem do seu estado de coinfecção e, portanto, não estão sendo tratadas recebendo os devidos cuidados em saúde (MINISTÉRIO DA SAÚDE, 2018a; UNAIDS, 2020; OMS, 2020). 
No município, 387 casos de coinfecção TB/HIV (13,8\%) foram diagnosticados e notificados, sendo que $256(92 \%)$ já estavam previamente infectados. Destes, apenas 31 casos (8\%) foram novos diagnósticos de HIV, ou seja, a maioria dos pacientes já era sabidamente portador do vírus ou encontravam-se na fase Aids anteriormente ao diagnóstico de TB.

De acordo com os manuais de recomendação do MS e OMS, é obrigatoriedade do serviço público de saúde ofertar a realização do rastreamento para HIV por meio de teste rápido no momento do diagnóstico da TB. O exame não foi realizado em $17,5 \%$ dos casos, o que revela possível falha na avaliação do paciente ou falta de recursos materiais no local do diagnóstico, fato este que não deveria ser um empecilho, pois se fosse o caso, o paciente deveria ser orientado e transferido para realizar o exame em outra unidade (MINISTÉRIO DA SAÚDE, 2018a).

De acordo com os dados do IBGE, o percentual de pacientes com diagnóstico de TB testados para HIV aumentou de maneira acelerada. No ano de 2019 , cerca de $76,1 \%$ dos casos novos já possuíam conhecimento acerca do seu status perante infecção pelo HIV, onde $8,4 \%$ foram positivos. A região Sul do país apresentou os maiores percentuais de testagem para o HIV, bem como as maiores porcentagens de coinfecção TB-HIV, seguido pelos estados do Amazonas e Distrito Federal (MINISTÉRIO DA SAÚDE, 2020; MAGNO ES, et al., 2017).

Assim, esses dados nos mostram uma melhora no diagnóstico da coinfecção da TB com o HIV, fato que vai de encontro com orientações nacionais para a prevenção da TB em pacientes com HIV. Afinal, o diagnóstico deve ser realizado precocemente em toda a população e avaliação oportuna da ocorrência de TB (MINISTÉRIO DA SAÚDE, 2020).

$\mathrm{O}$ alcoolismo/etilismo e dependência química tiveram incidência elevada quando comparado às outras doenças estando presente em $20,5 \%$ e $20 \%$ dos casos notificados, sendo a segunda e terceira maior ocorrência, respectivamente. O consumo de álcool e tabaco predominante na idade produtiva dos indivíduos está associado à organização etária da população, ratificando os dados identificados na literatura (GARCIA LP e FREITAS LRSD, 2015). No entanto, evidenciou-se a associação entre a dependência química e tabagismo por indivíduos infectados pela tuberculose da raça/cor branca, enquanto a literatura infere que a mortalidade por TB é superior entre pardos e negros (FIORI NS, et al., 2016; GIROTI SKO, et al., 2010).

O número de alcoólatras entre pacientes tuberculosos é alto, sendo que a incidência de tuberculose entre etilistas é significativamente mais alta do que na população geral. Esses dados são respaldados neste estudo, visto que dentre os 2068 casos de TB estimou-se uma taxa de $20,5 \%$ de alcoólatras (GARCIA LP e FREITAS LRSD, 2015).

O etilismo torna-se um importante fator de risco ao passo que interfere diretamente e indiretamente na infecção, tanto no contágio como na evolução da doença sendo um preditor de abandono, com baixa adesão ou uso irregular das medicações, que influenciam negativamente no tratamento com consequente risco de multirresistência, geralmente em níveis de dependência grave ao álcool. Além disso, há risco associado de intoxicação por drogas ilícitas, outro fator de risco (GIROTI SKO, et al., 2010; COUTO DS, et al., 2014).

O alcoolismo está associado a determinantes sociais relevantes, como ausência de moradia fixa, baixo status socioeconômico e desnutrição. Essa percentagem alta de etilistas entre os doentes poderia ser explicado pelo risco aumentado de infecção e uma baixa resistência à mesma, endógena e exógena, afinal os etilistas crônicos apresentam imunossupressão por deficiência proteico-calórica e de vitaminas decorrentes da má alimentação, tornando o hospedeiro suscetível à infecção pelo bacilo e ao desenvolvimento da doença (SILVA VD, et al., 2017; SAN PEDRO A e OLIVEIRA RM, 2013).

$O$ uso de drogas ilícitas aumenta o risco à infecção por TB em três vezes quando comparados àqueles que não as utilizam. O mecanismo é explicado pela redução da imunidade induzida por essas drogas e pelo estado físico característico de vulnerabilidade decorrente da dependência química, especialmente a má alimentação e má higiene, atrelada ao maior risco de disseminação do agente transmissor da TB nos locais de consumo, que são espaços notadamente pequenos, ambientes restritos e de condições urbanas precárias, onde há baixa circulação de ar e tendência à aglomeração (CASSIANO JGM, 2014; HALPERN SC, et al., 2017). 
Além disso, os usuários dessas substâncias representam um empasse no controle da TB. Esses indivíduos estão propensos a procurar atendimento em saúde em estágios já avançados da doença, omitindo o diagnóstico ou mesmo não dando a devida importância à necessidade de cuidados médicos, muitas vezes por conta dos efeitos de agitação psicomotora e redução do nível de consciência ocasionados pelas drogas. Isso leva à baixa adesão ao tratamento e perpetuação da cadeia de transmissão, em nível familiar e comunitário (CASSIANO JGM, 2014; HALPERN SC, et al., 2017; SILVA M, et al., 2017).

Apesar do Tratamento Diretamente Observado (TDO) ser uma abordagem importante no tratamento de TB, principalmente em pacientes alcoólicos e usuários de substâncias psicoativas devido a principalmente a dificuldade de adesão não foi objetivo da pesquisa, os dados obtidos apontam para a necessidade de avaliar o TDO no município (MINISTÉRIO DA SAÚDE, 2019b).

No ano de 2016 no Brasil, 36,2\% dos casos novos de TB pulmonar tiveram o seu tratamento na modalidade TDO, cujo percentual de cura comprovado laboratorialmente foi de 73,0\%31. O município de Campo Grande no mesmo ano foi a capital com menor percentual de cura comprovada laboratorialmente em relação ao país, com apenas 10,9\%. A capital líder de cura foi Macapá, com $86,1 \%$, sendo que os estados que atingiram os maiores percentuais foram Acre $(84,2 \%)$ e São Paulo $(81,6 \%) 31$. Este percentual baixo observado nessa pesquisa pode apontar problemas de gerenciamento do programa e atualização das informações no SINAN (MINISTÉRIO DA SAÚDE, 2018b; DATASUS, 2021).

A incidência predominante do tabagismo, alcoolismo, SPA e HIV/AIDS é respaldada na literatura e dialoga com fatores de risco para o adoecimento e falha no tratamento, seja pelas doenças, negligência, maior incidência no sexo masculino e nas penitenciárias, e aponta para a necessidade dos serviços de saúde implementarem suas ações de prevenção, diagnóstico e tratamento adequados desses fatores de risco e comorbidades, não só ao diagnosticar e sim anteceder ao adoecimento à TB, além da necessidade de reestruturar o conjunto de ações articuladas na rede de saúde que visem implementar o diagnóstico precoce da tuberculose nas unidades básicas de saúde, ações essas fundamentais para que se obtenha a queda da incidência da doença.

\section{CONCLUSÃO}

Os resultados deste estudo demonstraram incidência elevada de coinfecção por HIV e uso de drogas lícitas e ilícitas entre os casos de TB, reforçando a doença como um importante problema de saúde pública, agravada pelas comorbidades apresentadas. Observou-se possível ocorrência de atraso do diagnóstico, pois um terço dos casos de TB foram diagnosticados nos hospitais, além de ter ocorrido um coeficiente de incidência no município superior ao do estado de Mato Grosso do Sul e do Brasil, com um coeficiente de incidência de 51,42 e 41,6 casos/100 mil habitantes, respectivamente, em 2018 e 2019. Estes achados respaldam a importância da pesquisa para a área da saúde preventiva e coletiva, necessidade de mais investigações no estado e no país.

\section{REFERÊNCIAS}

1. AMICOSANTE MA, et al. Uso atual e aceitabilidade de novos testes diagnósticos para tuberculose ativa: um inquérito mundial. Jornal Brasileiro de Pneumologia, 2017; 43(5): 380-392.

2. CASSIANO JGM. Tuberculose pulmonar e o uso de drogas ilícitas: entre a cura e o abandono. Dissertação - Programa de Pós-Graduação em Enfermagem. Universidade Federal do Ceará, Fortaleza, 2014; 92 p.

3. COUTO DS, et al. Fatores determinantes para o abandono do tratamento da tuberculose: representações dos usuários de um hospital público. Saúde e Debate, 2014; 38(102): 572-81.

4. DEPARTAMENTO DE INFORMÁTICA DO SUS (DATASUS). População residente - estudo de estimativas populacionais por município, idade e sexo 2000-2020, Brasil, 2021. Disponível em: <http://tabnet.datasus.gov.br/cgi/deftohtm.exe?popsvs/cnv/popbr.def>. Acessado em 22 de fevereiro de 2021.

5. DEPARTAMENTO DE INFORMÁTICA DO SUS (DATASUS). Tuberculose - casos confirmados notificados no sistema de informação de agravos de notificação - Brasil: Período de 2001 a 2019, Brasil, 2021. Disponível em: <http://tabnet.datasus.gov.br/cgi/tabcgi.exe?sinannet/cnv/tubercbr.def>. Acessado em 22 de fevereiro de 2021.

6. DEPARTAMENTO DE INFORMÁTICA DO SUS (DATASUS). Informações de saúde (TABNET) - Demográficas e socioeconômicas. Brasil, 2021. Disponível em: <http://www2.datasus.gov.br/DATASUS/index.php?area=0206>. Acessado em 22 de fevereiro de 2021. 
7. FIORI NS, et al. Prevalência e fatores associados ao tabagismo em fumicultores do Sul do Brasil. Cadernos de Saúde Pública, 2016, 32(7).

8. FREITAS VMT, et al. Perfil clínico-epidemiológico de pacientes portadores de tuberculose atendidos em uma unidade municipal de saúde de Belém, Estado do Pará, Brasil. Revista Pan-Amazônica da Saúde, 2016, 7(2): 45-50.

9. GARCIA LP, FREITAS LRSD. Consumo abusivo de álcool no brasil: resultados da pesquisa nacional de saúde 2013. Epidemiologia e Serviços de Saúde, 2015; 24(2): 227-237.

10. GASPAR RS, et al. Análise temporal dos casos notificados de tuberculose e de coinfecção tuberculose HIV na população brasileira no período entre 2002 e 2012. Jornal Brasileiro de Pneumologia, 2016; 42(6): 416-422.

11. GIROTI SKO, et al. Perfil dos pacientes com tuberculose e os fatores associados ao abandono do tratamento. Cogitare Enfermagem, 2010; 15(2): 271-7.

12. HALPERN SC, et al. Vulnerabilidades clínicas e sociais em usuários de crack de acordo com a situação de moradia: um estudo multicêntrico de seis capitais brasileiras. Cadernos de Saúde Pública, 2017; 33(6).

13. INSTITUTO BRASILEIRO DE GEOGRAFIA E ESTATÍSTICA (IBGE). Estimativas da população residente com data de referência 10 de julho de 2020. Disponível em: <https://cidades.ibge.gov.br/brasil/ms/campo-grande/panorama>. Acessado em 22 de fevereiro de 2021.

14. JOINT UNITED NATIONS PROGRAMME ON HIV/AIDS (UNAIDS). Global Report: UNAIDS Data 2020 - Report on the Global AIDS Epidemic. Geneva, 2020. Disponível em: https://www.unaids.org/en/resources/documents/2020/globalaids-report. Acessado em 22 de fevereiro de 2021.

15. MAGNO ES, et al. Fatores associados à coinfecção tuberculose e HIV: o que apontam os dados de notificação do Estado do Amazonas, Brasil, 2001-2012. Cadernos de Saúde Pública, 2017; 33(5).

16. MINISTÉRIO DA SAÚDE. Plano Nacional pelo Fim da Tuberculose como Problema de Saúde Pública. Brasília: Brasil, 2017. Disponível em: https://bvsms.saude.gov.br/bvs/publicacoes/brasil_livre_tuberculose_plano_nacional.pdf. Acessado em 22 de fevereiro de 2021.

17. MINISTÉRIO DA SAÚDE. Secretaria de Vigilância em Saúde, Departamento de Vigilância, Prevenção e Controle das Infecções Sexualmente Transmissíveis, do HIV/Aids e das Hepatites Virais. Protocolo Clínico e Diretrizes Terapêuticas para Manejo da Infecção pelo HIV em Adultos. Brasília: Brasil, 2018a. 412 p.

18. MINISTÉRIO DA SAÚDE. Secretaria de Vigilância em Saúde. Boletim Epidemiológico. Brasil, 2018b, 49(11). Disponível em: <https://portalarquivos2.saude.gov.br/images/pdf/2018/marco/26/2018-009.pdf>. Acessado em 22 de fevereiro de 2021.

19. MINISTÉRIO DA SAÚDE. Secretaria de Vigilância em Saúde. Boletim Epidemiológico. Brasília, vol. 50, n. 09, 2019a. Disponível em: https://portalarquivos2.saude.gov.br/images/pdf/2019/marco/22/2019-009.pdf. Acessado em 22 de fevereiro de 2021.

20. MINISTÉRIO DA SAÚDE. Secretaria de Vigilância em Saúde. Departamento de Vigilância Epidemiológica. Manual de recomendações para o controle da tuberculose no Brasil. Ministério da Saúde. 2019b. Brasil, 2019b. Disponível em: https://bvsms.saude.gov.br/bvs/publicacoes/manual_recomendacoes_controle_tuberculose_brasil_2_ed.pdf.Acessad o em 22 de fevereiro de 2021.

21. MINISTÉRIO DA SAÚDE. Secretaria de Vigilância em Saúde. Boletim Epidemiológico - Tuberculose. Ministério da Saúde. Número Especial. Mar. Brasil, 2020. Disponível em: <https://antigo.saude.gov.br/images/pdf/2020/marco/24/Boletim-tuberculose-2020-marcas--1-.pdf>. Acessado em 22 de fevereiro de 2021.

22. NOVOTNY T, et al. HIV/AIDS, tuberculose e tabagismo no Brasil: uma sindemia que exige intervenções integradas. Cadernos de Saúde Pública, 2017; 33(3).

23. OLIVEIRA LGF, et al. Incidência da tuberculose na Bahia: o retrato de uma década. REVISE - Revista Integrativa em Inovações Tecnológicas nas Ciências da Saúde, 2021; 4(00).

24. OLIVEIRA MG, et al. Anemia em pacientes internados com tuberculose pulmonar. Jornal Brasileiro de Pneumologia, 2014; 40(4): 403-410.

25. OROFINO RL, et al. Preditores dos desfechos do tratamento da tuberculose. Jornal Brasileiro de Pneumologia, 2012; 38(1): 88-97.

26. ORME IM, et al. The balance between protective and pathogenic immune responses in the TB-infected lung. National Immunology, 2015; 16(1): 57-63.

27. PEREIRA SM, et al. Associação entre diabetes e tuberculose: estudo caso controle. Revista de Saúde Pública, 2016; $50(82)$.

28. SAN PEDRO A, OLIVEIRA RM. Tuberculose e indicadores socioeconômicos: revisão sistemática da literatura. Revista Panamericana Salud Pública, 2013; 33(4): 294-301.

29. SILVA DR, et al. Fatores de risco para tuberculose: diabetes, tabagismo, álcool e uso de outras drogas. Jornal Brasileiro de Pneumologia, 2018; 44(2): 145-152.

30. SILVA M, et al. Drug addiction and alcoholism as predictors for tuberculosis treatment default in Brazil: a prospective cohort study. Epidemiology and Infection, 2017; 145(16): 3516-3524.

31. SILVA VD, et al. Estimativas das taxas de recidiva, cura e abandono de tratamento em pacientes com tuberculose pulmonar tratados com esquema de dose fixa combinada de quatro drogas em uma unidade de saúde terciária na cidade do Rio de Janeiro. Jornal Brasieliro de Pneumologia, 2017; 43(2): 113-20.

32. VALENÇA MS, et al. Tuberculose em presídios brasileiros: uma revisão integrativa da literatura. Ciência e Saúde Coletiva, 2016; 21(7): 2147-2160.

33. ORGANIZAÇÃO MUNDIAL DA SAÚDE (OMS). Global Tuberculosis Report 2020. Geneva, 2020. Disponível em: https://www.who.int/publications/i/item/9789240013131/. Acessado em 22 de fevereiro de 2021. 